\title{
Dashboard system for measuring green software design
}

\begin{abstract}
Global ICT industry is claimed to account for approximately 2 percent of global carbon dioxide which is a figure equivalent to the aviation industry. This is due to lack of awareness to reduce the environmental impact of ICT products and services by the ICT industry practitioners. Therefore, we proposed a GreenDash system, a web-based dashboard that provides a platform for software developers to measure green software design. Software developers capable to calculate sustainability of green software design based on the four components of Information System (IS): database, hardware, people and network. GreenDash System helps to assist software developers to ensure that they considered about green software in designing software product and services.
\end{abstract}

Keyword: Green software design; Dashboard system; Green design 